\title{
Estudo morfométrico da terapia LED de baixa potência em tendinite de ratos
}

\author{
Morphometric study of low power LED therapy tendonitis in rats
}

José Mário Nunes da Silva ${ }^{1}$, Janderson Pereira de Carvalho ${ }^{2}$, Manoel de Jesus Moura Júnior ${ }^{3}$

Estudo desenvolvido no

Laboratório de Fisiologia da Faculdade Santo Agostinho (FSA) como parte da dissertação de mestrado "Estudo por FT - Raman da ação do LED de baixa potência e do ultrassom terapêutico em modelo experimental de tendinite em ratos".

1 Fisioterapeuta; Bacharelando em Estatística pela Universidade Federal do Piauí (UFPI) - Teresina (PI), Brasil.

2 Fisioterapeuta graduado na FSA Teresina (PI), Brasil.

${ }^{3}$ Mestre em Engenharia Biomédica pela Universidade Vale do Paraíba (UNIVAP); Coordenador de Fisioterapia da FSA - Teresina (PI), Brasil.

\section{ENDEREÇO PARA CORRESPONDÊNCIA} José Mário Nunes da Silva - Rua 8, 6.209 - Residencial Primavera Leste CEP: 64057-180 - Teresina (PI), Brasil -E-mail: zemariu@hotmail.com

\section{APRESENTAÇÃO}

mar. 2011

\section{ACEITO PARA PUBLICAÇÃO}

out. 2011

\section{FONTE DE FINANCIAMENTO} nenhuma

\section{CONFLITO DE INTERESSE}

\section{nada a declarar}

Aprovação do Comitê de Ética da Faculdade Integral Diferencial (FACID) com parecer favorável $\mathrm{n}^{\circ}$ 086/09
RESUMO: A terapia LED de baixa potência possui efeitos analgésico e antiinflamatório. O objetivo desse estudo foi analisar a ação da terapia com LED de baixa potência na reparação tendínea por meio de histologia e histomorfometria. Foram usados 25 ratos Wistar, de 220 a $250 \mathrm{~g}$, divididos em três grupos experimentais avaliados no $7^{\circ}$ e $14^{\circ}$ dia: $A$, tendinite induzida sem tratamento; $B$, tendinite induzida tratada com LED de baixa potência, densidade de energia 4 J/cm² por 120 segundos; e C, sem indução de tendinite. A histomorfometria mostrou-se altamente significativa $(p<0,001)$ na terapia com LED em contrarrelógio de 14 dias, comparada ao grupo saudável (C). Entretanto, não mostrou significância levando em conta o grupo tendinite (A14), em que valor $p>0,05$. As análises histológica e histomorfométrica demonstraram qualitativo e quantitativo aumento no número de fibroblastos aos sete dias de tratamento, e das fibras colágenas, aos 14 dias, para grupo tratado com LED de baixa potência em relação ao grupo sadio $(\mathrm{C})$. O mesmo não foi percebido quando relacionado ao grupo tendinite (A14).

Descritores: inflamação; tendinopatia; fototerapia.

ABSTRACT: The low power LED therapy has analgesic and anti-inflammatory effects. The objective of this study was to analyze the action of therapy with low power LED on the tendon repair, using histology and histomorphometry. Were used 25 Wistar rats, with 220 to 250 g, divided into three experimental groups ( 7 and 14 days): A7 and A14 induced tendonitis without treatment; B7 and B14, induced tendinitis treated with LED low power density energy $4 \mathrm{~J} / \mathrm{cm}^{2}$ for 120 seconds; and C, without induction of tendinitis. Histomorphometry was highly significant $(p<0.001)$ with LED therapy against the clock for 14 days, compared with the healthy group (C). But not significant compared with the tendinitis group (A14) ( $p>0.05)$. Histology and histomorphometry analysis demonstrated qualitative and quantitative increase in the number of fibroblasts to seven days of treatment, and collagen fibers at 14 days for the group treated with LED low power compared to the healthy group (C). But it is unclear even when related to the tendinitis group (A14).

KeYwords: inflammation; tendinopathy; phototherapy. 


\section{INTRODUCÃO}

A tendinite é uma lesão de esforço, na maioria das vezes causada pela realização do mesmo movimento repetidamente $^{1}$. A inflamação aguda é condição desencadeante do processo de reparação tecidual que, normalmente, é exagerado e pode acarretar complicações, prejudicando a qualidade do tecido formado ${ }^{2}$.

Lesões do tendão são comuns no esporte, e estima-se que representem de 30 a $50 \%$ das ocorrências nesse tipo de atividade. Sua incidência anual em corredores de alto nível é entre 7 e $9 \%{ }^{2-4}$. Além disso, a tendinite é um dos principais problemas relacionados à lesão por esforço repetitivo e a distúrbios osteomusculares vinculados ao trabalho'.

Com a finalidade de amenizar e curar essa afecção, novos métodos de diagnóstico e tratamento estão sendo introduzidos. Atualmente existem no mercado recursos que utilizam a luz como terapia, dentre os quais podemos citar Light Emitting Diode (LED's) e Light Amplification by Stimulated Emission of Radiation (LASER's). Eles estimulam a aceleração da cicatrização de partes moles, com resultados satisfatórios no processo de regeneração do tendão ${ }^{5-9}$.

A principal diferença da luz emitida pelo LED em relação à emitida por um laser é a ausência de coerência ${ }^{1,5}$. Entretanto, a coerência da luz não é responsável pelos efeitos de terapias com laser de baixa potência, pois esta propriedade se perde nas primeiras camadas de tecido biológico ${ }^{10-12}$.

A razão do aumento de pesquisas buscando comparar os efeitos da fototerapia com o uso de LEDs em substituição ao laser é que, diferente do comprimento de onda, a sua coerência não é um fator importante para o efeito biomodulador. Além disso, os aparelhos que emitem radiação laser têm alto custo, fazendo-se necessária a busca por fontes luminosas alternativas. Nesse contexto, os LED's surgem como uma excelente opção em virtude do seu potencial de ação e de seu valor mais barato ${ }^{6,13}$. Estudos de Karu et al. ${ }^{8}$ registraram resultados clínicos satisfatórios sob o aspecto das diferentes fontes de luz, sugerindo efeitos benéficos advindos da terapia com LED em diversos tipos de lesões cutâneas.
Porém, existem discordâncias de pesquisadores quanto à eficiência dessa terapia na biomodulação tecidual. Enquanto alguns estudos demonstram efeitos como inibidor da sintomatologia dolorosa ou aceleração de reparo de lesões cutâneas ${ }^{6-8,10,13}$, outros apresentam a interação da biomodulação LED na reparação tendínea.

A presente pesquisa foi realizada devido à escassez de trabalhos sobre o uso da terapia LED de baixa potência na reparação tendínea e pela necessidade de terapias alternativas para auxílio deste processo.

\section{MÉTODOLOGIA}

Todos os experimentos foram realizados de acordo com as orientações para o cuidado animal, seguindo as premissas do Colégio Brasileiro de Experimentação Animal (COBEA). A pesquisa foi aprovada pelo Comitê de Ética, sob o protocolo $\mathrm{n}^{\circ}$ 086/09. Foram utilizados 25 ratos (Rattus norvegicus, albinus), linhagem Wistar entre 30 e 60 dias de idade, com peso médio de $230 \mathrm{~g}$.

\section{A indução da tendinite}

Os animais foram anestesiados com ketamina $(100 \mathrm{mg} / \mathrm{kg}$, ip) e xilazina (20 mg/kg, p) e, posteriormente, submetidos à indução da tendinite. Para induzir experimentalmente a tendinite, aplicouse uma injeção intratendínea de $10 \mu \mathrm{L}$ de colagenase (10 mg/mL; SIGMA; C6885) no tendão do calcâneo direito dos ratos, seguindo estudos de Silva et al. ${ }^{14}$. Os animais receberam a injeção de colagenase no primeiro dia e foram sacrificados com dose excessiva de anestésico (tiopental), 7 e 14 dias após a indução.

\section{Intervenção terapêutica experimental}

O tratamento de cada animal teve início 24 horas após a indução da tendinite, sendo mantido a cada 24 horas depois da primeira aplicação da terapia com LED. Os ratos foram posicionados em decúbito ventral com imobilização manual da pata direita para aplicação da terapêutica indicada em contato direto com a área a ser tratada. A aplicação da terapia se deu de forma pontual, na qual o feixe emitido cobria por inteiro o sítio da lesão. O aparelho foi posicionado a $90^{\circ}$ do eixo tendíneo com intenção de otimizar a aplicação, evitando perdas de energia e possibilitando melhor contato com a perna do animal.

\section{Protocolo de tratamento e grupos experimentais}

Os animais foram divididos em grupos experimentais com cinco indivíduos cada, conforme descrito na Tabela 1.

Após a indução experimental da tendinite, os animais do grupo A7 e A14 (tendinite) foram mantidos em gaiolas, sem qualquer tratamento. Já os do B7 e B14 (LED) receberam diariamente terapia com LED de baixa potência (Tabela 2). O grupo C (sadio) foi composto pelos tendões da perna esquerda dos animais de A7 e A14, seguindo as premissas do COBEA.

\section{Preparação da amostra}

Todos os cinco animais de cada grupo foram sacrificados 7 ou 14 dias após a indução experimental de tendinite. Os tendões foram removidos por dissecção dos tecidos da perna direita. Os ratos dos grupos A7 e A14 tiveram os tendões da pata esquerda dissecados para serem analisados e comporem o grupo sadio (C).

\section{Análise histológica}

Os tendões foram seccionados, longitudinalmente desidratados em soluções crescentes de álcool e diafanizados em xilol, incluídos, em seguida, em parafina. Obtiveram-se quatro cortes semisseriados

Tabela 1. Divisão dos grupos experimentais

\begin{tabular}{lccc}
\hline $\begin{array}{l}\text { Grupos } \\
(\mathrm{n}=15)\end{array}$ & $\begin{array}{c}\text { Subgrupos } \\
(\mathrm{n}=5)\end{array}$ & $\begin{array}{c}\text { Indução da } \\
\text { tendinite }\end{array}$ & Tratamento \\
\hline A & A7 & Sim & Não \\
B & A14 & Sim & Não \\
& B7 & Sim & LED \\
C & B14 & Sim & LED \\
\hline
\end{tabular}


Tabela 2. Parâmetros de tratamento utilizados

\begin{tabular}{ll}
\hline Equipamento & LED de baixa potência \\
\hline Pico de banda & $640 \mathrm{~nm}$ \\
Potência média & $30 \mathrm{~mW}$ \\
Spot & $0,5 \mathrm{~cm}^{2}$ \\
Densidade de energia & $4 \mathrm{~J} / \mathrm{cm}^{2}$ \\
Disparo & Manual \\
Tempo & $120 \mathrm{seg}$ \\
\hline
\end{tabular}

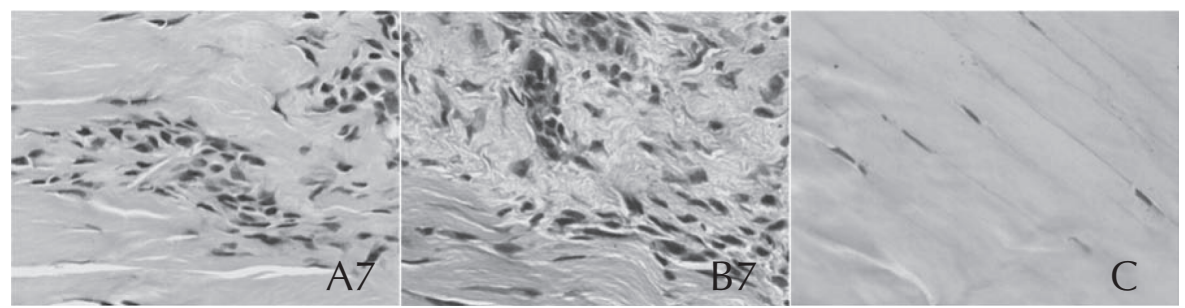

Figura 1. Aspectos histológicos do tendão dos animais dos grupos experimentais A7, grupo tendinite, sete dias; B7 grupo LED, sete dias; e C, grupo sadio

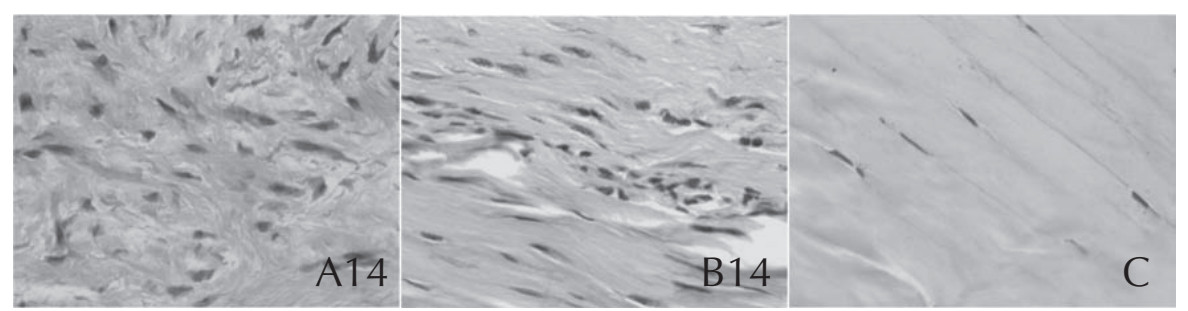

Figura 2. Aspectos histológicos do tendão dos animais dos grupos experimentais A14, grupo tendinite, 14 dias; B14 grupo LED, 14 dias; e C, grupo sadio

com $5 \mu \mathrm{m}$ de espessura, sendo dois corados com hematoxilina-eosina (HE) e dois pelo Tricrômico de Masson (TM), que permite visualizar as fibras colágenas. $\mathrm{O}$ material foi examinado com um microscópio óptico trinolucular Olympus CX31, modelo YS100, equipado com câmera digital Olympus SC20 e acoplado a um microcomputador.

\section{Avaliação histomorfométrica}

Foi realizada nas lâminas coradas com o TM, sendo quantificado o número de fibroblastos. Sob aumento de 400 vezes, capturaram-se de cada lâmina dois campos microscópicos nos quais havia maior concentração celular. As imagens digitalizadas foram avaliadas com o auxílio do programa de análise computacional de imagens Image- $\rfloor^{\circledR}$ (Versão 1,32 para Windows). do que no $14^{\circ}$ dia do tratamento. No grupo tendinite (A7), havia espessamento do epitendão e endotendão. Observouse também a formação de fibroblastos volumosos, com citoplasma abundante (Figura 1). Por sua vez, os espécimes que compunham o grupo tratado com LED de baixa potência (B7) encontravam-se com epitendão e endotendão, tecido de granulação abundante, caracterizado por fibroblastos e vasos sanguíneos neoformados. Em comparação com o grupo tendinite (A7), os fibroblastos eram aparentemente mais numerosos, dispondo-se de forma mais organizada (Figura 1).

No grupo tendinite (A14), as células inflamatórias mononucleadas e os vasos neoformados no epitendão e endotendão aparentemente reduziram, enquanto o número de fibroblastos continuou elevado. No grupo tratado com LED de baixa potência (B14), os vasos tornaram-se bastante escassos e a matriz extracelular apresentou-se mais densa. Os fibroblastos, mais delgados e fusiformes, tinham características mais próximas de fibrócitos e se dispunham em feixes orientados em paralelo com os não danificados. Entretanto, em nenhum dos espécimes estudados os setores lesados chegaram a reconstruir a estrutura normal do tendão do grupo sadio (C) (Figura 2).

Nas amostras que compuseram o grupo saudável, os tendões normais demonstraram características histológicas, fibrócitos alongados, com pouco citoplasma e núcleo fusiforme, foram encontrados esparsamente entre feixes densos e paralelos de colágeno (Figuras $1 \mathrm{C}$ e $2 \mathrm{C}$ ).

Os dados foram coletados e avaliados quanto ao coeficiente de variação e à distribuição amostral para determinação do teste estatístico, considerando o nível de significância estatística de 5\% $(p<0,05)$. Realizou-se a análise estatística da variação do número de células inflamatórias, obtidas nos grupos tratados e não tratados, utilizando-se o teste de ANOVA, com pós-teste de Tukey, por meio do programa estatístico GraphPad Prism $^{\circledR}$, versão 3.0.

\section{RESULTADOS}

$\mathrm{Na}$ análise dos cortes histológicos foi encontrado, após sete dias de tratamento, um padrão histológico menos organizado
A histomorfometria resultante da análise histológica dos espécimes permitiu observar que no tempo experimental de sete dias os valores mostraram-se equivalentes nos grupos tendinite e tratado com LED terapia, sendo ambos maiores do que a quantidade de fibroblastos presentes no grupo sadio.

Ressalta-se que, durante a fase inicial do processo de reparo, as fibras colágenas recém-formadas por fibroblastos jovens e volumosos, presentes em grande número, apresentam-se imaturas, pouco espessas e com distribuição aleatória. Nessa fase ainda podemos visualizar áreas com tecido de granulação e algumas células inflamatórias. 




*p>0,050 não significante em relação ao grupo tendinite no tempo experimental de 14 dias; ${ }^{* *} p<0,001$, extremamente significante em relação ao grupo sadio no tempo experimental de 7 e 14 dias

Figura 3. Comparativo da variação $(\Delta)$ do número de fibroblastos após 7 e 14 dias de tratamento

A observação da Figura 3 nos permite notar a evolução do processo de reparo nos diferentes grupos experimentais depois de transcorridos 14 dias. A comparação dos resultados do que recebeu tratamento e do tendinite com os do sadio (C), quanto ao número de fibroblastos na área de lesão, foram estatisticamente significantes. Por outro lado, os resultados comparativos do grupo LED (B14) e tendinite (A14) não foram estatisticamente significantes.

\section{DISCUSSÃO}

A presente pesquisa demonstrou, por meio de análise histológica e histomorfométrica, um melhor efeito próinflamatório no grupo tratado com LED em relação ao grupo tendinite.

Concordando com os resultados obtidos, Silva ${ }^{15}$ utilizou modelo experimental de indução de tendinite semelhante ao empregado neste estudo, e relatou que a evolução do processo de reparo, analisado pela espectrometria Raman, ocorreu com etapas bem características. Os resultados dos grupos tratados mostraram que o tratamento com LED induziu proliferação fibroblástica. Concordando com esses achados, Casalechi ${ }^{16}$ registrou diminuição significativa do número de

confirmado por Houreld ${ }^{19}$ que observou
$5 \mathrm{~J} / \mathrm{cm}^{2}$, enquanto as células que receberam dose de $16 \mathrm{~J} / \mathrm{cm}^{2}$ apresentaram efeito inibitório na proliferação e atividade do fibroblasto.

Diante dos resultados é possível afirmar que muitos dos achados reportados por outros autores são similares aos deste estudo, o que nos permite verificar que os resultados obtidos pela fotobiomodulação, com a utilização de uma fonte emissora de luz não coerente, são semelhantes aos obtidos com o uso dos vários tipos de laser de baixa potência, os quais possuem coerência, mas que se perdem com a profundidade do tecido ${ }^{16}$.

Faria $^{20}$ comparou a utilização do LED com um laser de baixa potência (sem variação de parâmetros) e observou maior eficácia do LED na reparação tecidual de tecido tendinoso em comparação ao laser. Segundo Casalechi ${ }^{16}$, pode-se observar que os efeitos do LED na prática experimental apresentam eficácia no processo de reparação tecidual de tendões.

Contudo, torna-se necessário o conhecimento sobre a dose e os mecanismos que influenciam na recuperação funcional dos tendões reparados com LED, pois o estabelecimento de protocolos adequados poderá auxiliar mais rapidamente na incorporação desse tipo de fototerapia na prática clínica-fisioterapêutica.

Estudos demonstram ainda que a utilização da terapia LED de baixa potência pode ser eficiente para prevenção da geração de cáries, com efeito bactericida, alívio da dor na cicatrização ${ }^{21-23}$ e reparação óssea ${ }^{24,25}$, demonstrando a versatilidade da aplicação do LED. Além disso, abre novas perspectivas para futuros estudos de associação, pois a sua aplicação parece apresentar efeitos benéficos para a tendinite. Os resultados distintos encontrados podem ter ocorrido em consequência da utilização de diferentes parâmetros na aplicação do LED, o que torna necessária a realização de mais estudos na área para estabelecer um protocolo de tratamento no seu uso.

\section{CONCLUSÃO} o efeito estimulante da terapia com LED nas células que receberam dose igual a tudo pode ter sido um fator na limitação da redução do número de fibroblastos no tempo do experimento de 14 dias, pois segundo Almeida et al. ${ }^{18}$, ela pode influenciar o crescimento celular. Isto foi
As análises histológica e histomorfométrica revelaram que os espécimes 
tratados com LED apresentaram maior ação pró-inflamatória quando comparados ao grupo sadio (C) no tempo experimental de 14 dias. Também demonstraram aumento qualitativo e quantitativo no número de fibroblastos aos 7 dias de tratamento, e das fibras colágenas aos 14 dias para grupo tratado com LED em relação ao grupo sadio (C). Não foi percebido o mesmo quando se comparou aos grupos tendinite (A14).

\section{REFERENNCIAS}

1. Maffulli N, Kader D. Tendinopathy of tendo achillis. J Bone Joint Surg Br. 2002;84(1):1-8.

2. Paavola M, Kannus $P$, Järvinen TA, Khan K, Józsa L, Järvinen M. Achilles tendinopathy. J Bone Joint Surg Am. 2002;84-A(11):2062-76.

3. Maffulli N, Ewen SW, Waterston SW, Reaper J, Barrass $\mathrm{V}$. Tenocytes from ruptured and tendinopathic achilles tendons produce greater quantities of type III collagen than tenocytes from normal achilles tendons. An in vitro model of human tendon healing. Am J Sports Med. 2000;28(4):499-505.

4. Covizi DZ, Felisbino SL, Gomes L, Pimentel ER, Carvalho HF. Regional adaptations in three rat tendons. Tissue Cell. 2001;33(5):483-90.

5. Andrade MF, Rastelli ANS, Saad RS, Saad JRC. Avaliação da capacidade de polimerização de um novo dispositivo a base de LED à bateria. J Am Dent Assoc. 2001;4:372-76.

6. Corazza AV. Fotobiomodulação comparativa entre o Laser e LED de baixa intensidade na angiogênese de feridas cutâneas de ratos. 2005. Dissertação (Mestrado em Bioengenharia) - Escola de Engenharia de São Carlos; Faculdade de Medicina de Ribeirão Preto; Instituto de Química de São Carlos, Universidade de São Paulo, São Paulo; 2005.

7. Mavropoulos A; Staudt CB; Kiliaridis S; Krejci I. Light curing time reduction: in vitro evaluation of new intensive light-emitting diode curing units. Eur J Orthod. 2005;27(4):408-12.

8. Karu TI; Kolyakov SF. Exact action spectra for cellular responses relevant to phototherapy. Photomed Laser Surg. 2005;23(4):355-61.

9. Pacheco MTT. Laseres Noções Básicas. São José dos Campos: UNIVAP - Universidade do Vale do Paraíba; 2007.

10. Boulton M, Marshall J. He-Ne laser simulation of human fibroblast proliferation and attachment in vitro. Lasers in the Life Sciences. 1986;1:125-34.

11. Pontinen P. Laseracupunture. In: Simunovie Z, editor. Lasers in Medicine and Dentistry. Part One: Basic Science, and Up-to-date Clinical Application of Low Energy-Laser Laser Therapy LLLT. 1 ed. Rijeka: Vitgraf; 2000. p.455-475.
12. Whelan HT, Houle JM, Whelean NT, Donohoe DL, Cwiklinski J, Shmitd MH, et al.. The NASA LightEmitting Diode medical program- Progress in space flight terrestrial applications. Space Tech App Int Forum; 2000;504:37-43.

13. Sacono NT, Costa CA, Bagnato VS, Abreu-e-Lima FC. Light-emitting diode therapy in chemotherapy-induced mucositis. Lasers Surg Med. 2008;40(9):625-33.

14. Silva JMN, Carvalho JP, Moura Júnior MJ, Arisawa EALS, Martin AA, Sá HP, et al. Estudo da ação do ultrassom terapêutico em modelo experimental de tendinite em ratos Wistar. Conscientiae Saúde; 2010;9(4):625-32.

15. Silva EJ. Espectroscopia Raman e histologia clássica na avaliação de tendinite induzida por colagenase em ratos Wister. 2005. Ddissertação. Franca: Universidade de Franca, São Paulo; 2005.16. Casalechi HL. Efeito da terapia com LED de baixa potência sobre o processo de reparação do tendão de Aquiles em ratos. 2008. Ddissertação. São José dos Campos: Universidade do Vale do Paraíba, São Paulo; 2008.

17. Vinck EM, Cagnie BJ, Cornelissen MJ, Declercq HA, Cambier DC. Increased fibroblast proliferation induced by light emitting diode and low power laser irradiation. Lasers Med Sci. 2003;18(2):95-9.

18. Almeida-Lopes L, Rigau J, Zângaro RA, GuidugliNeto J, Jaeger MM. Comparison of the low level laser therapy effects on cultured human gingival fibroblasts proliferation using different irradiance and same fluence. Lasers Surg Med. 2001;29(2):179-84.

19. Houreld NN, Abrahamse H. Laser light influences cellular viability and proliferation in diabetic-wounded fibroblast cells in a dose- and wavelength-dependent manner. Lasers Med Sci. 2006;23(1):11-8.

20. Faria ETB. Estudo dos efeitos da terapia com luz coerente e não coerente sobre a reparação tendínea através da espectroscopia Raman e análise histopatológica. 2006. Dissertação (Mestrado em Bioengenharia). São José dos Campos: Instituto de Pesquisa e Desenvolvimento da Universidade do Vale do Paraíba, São Paulo; 2006.

21. Bevilacqua IM; Nicolau RA; Khouri S; Brugnera AJR; Teodoro GR; Zângaro RA, et al. The impact of photodynamic therapy on the viability of Streptococcus mutans in a planktonic culture. Photomed Laser Surg. 2007;25(6):513-8. 


\section{Referências (cont.)}

22. Breitenbach S; Nicolau RA; Nóbrega CJP. Terapia LED de baixa intensidade no tratamento da dor durante movimento ortodôntico - Estudo Clínico. ConScientiae Saúde. 2010;9(4):684-92.

23. Tortamano A, Lenzi DC, Haddad AC, Bottino MC, Dominguez GC, Vigorito JW. Low-level laser for pain caused by placement of the first orthodontic archwire: a randomized clinical trial. Am J Orthod Dentofacial Orthop. 2009;136(5):662-7.
24. Araújo KS. Efeito da Terapia com LED operando em baixa potência sobre a reparação óssea de ratos - análise por espectroscopia Raman. 2009. Ddissertação (Mestrado em Bioengenharia). São José dos Campos: Instituto de Pesquisa e Desenvolvimento da Universidade do Vale do Paraíba, São Paulo; 2009.

25. Pinheiro AL, Limeira Júnior FA, Gerbi ME, Ramalho LM, Marzola C, Ponzi EA. Effect of low level laser therapy on the repair of bone defects grafted with inorganic bovine bone. Braz Dent J. 2003;14(3):177-81. 